\title{
Characterization of the Fungal Microbiome (Mycobiome) in Fecal Samples from Dogs
}

\author{
M. Lauren Foster, ${ }^{1}$ Scot E. Dowd, ${ }^{2}$ Christine Stephenson, ${ }^{1}$ \\ Jörg M. Steiner, ${ }^{1}$ and Jan S. Suchodolski ${ }^{1}$ \\ ${ }^{1}$ Gastrointestinal Laboratory, Department of Small Animal Clinical Sciences, College of Veterinary Medicine \& Biomedical Sciences, \\ Texas A\&M University, College Station, TX 77843, USA \\ ${ }^{2}$ Molecular Research DNA Laboratory, Shallowater, TX 79363, USA
}

Correspondence should be addressed to Jan S. Suchodolski; jsuchodolski@cvm.tamu.edu

Received 21 December 2012; Revised 4 April 2013; Accepted 5 April 2013

Academic Editor: Lorraine M. Sordillo

Copyright (C) 2013 M. Lauren Foster et al. This is an open access article distributed under the Creative Commons Attribution License, which permits unrestricted use, distribution, and reproduction in any medium, provided the original work is properly cited.

\begin{abstract}
The prevalence and phylogenetic description of fungal organisms and their role as part of the intestinal ecosystem have not yet been studied extensively in dogs. This study evaluated the fungal microbiome of 19 dogs (12 healthy dogs and 7 dogs with acute diarrhea) using fungal tag-encoded FLX-Titanium amplicon pyrosequencing. Five distinct fungal phyla were identified, with Ascomycota (medians: $97.9 \%$ of obtained sequences in healthy dogs and $98.2 \%$ in diseased dogs) and Basidiomycota (median $1.0 \%$ in healthy dogs and median $0.5 \%$ in diseased dogs) being the most abundant fungal phyla. A total of 219 fungal genera were identified across all 19 dogs with a median (range) of 28 (4-69) genera per sample. Candida was the most abundant genus found in both the diseased dogs (median: $1.9 \%$, range: $0.2 \%-38.5 \%$ of sequences) and healthy dogs (median: $5.2 \%$, range: $0.0 \%-63.1 \%$ of sequences). Candida natalensis was the most frequently identified species. No significant differences were observed in the relative proportions of fungal communities between healthy and diseased dogs. In conclusion, fecal samples of healthy dogs and dogs with acute diarrhea harbor various fungal genera, and their role in gastrointestinal health and disease warrants further studies.
\end{abstract}

\section{Introduction}

Recent molecular-phylogenetic studies have revealed diverse microbial communities in the canine gastrointestinal (GI) tract and have emphasized the importance of the intestinal microbiota for gastrointestinal health [1]. The intestinal microbiota plays a vital role in the health of the GI tract, participates in the development of the host immune system, and also provides protection from invading pathogens [2]. Several studies have characterized the bacterial communities in the canine GI tract in health and disease [3-5]. However, limited information is available about the prevalence and classification of other members of the intestinal microbiome, such as fungal organisms. Previous studies that have described fungal organisms in the canine GI tract have either used culture based methods [6,7], have utilized molecular-phylogenetic methods on pooled intestinal samples [8,9], or have analyzed only a limited number of fungal sequences [10]. Studies in humans have suggested that the fungal microbiome may play a role in chronic GI disorders [11, 12]. Therefore, a more detailed description of the fungal microbiome (mycobiome) is needed to better understand the role of fungi in the GI tract of healthy dogs and dogs with GI disease.

The aim of this study was to describe the fungal communities present in fecal samples obtained from healthy dogs and dogs with acute, nonhemorrhagic diarrhea using highthroughput $18 \mathrm{~S}$ rRNA gene pyrosequencing.

\section{Materials and Methods}

2.1. Fecal Samples. Naturally passed fecal samples were collected from a total of 19 privately owned dogs (12 healthy dogs and 7 dogs with acute diarrhea). All dogs, healthy and 
diseased, lived in Texas at the time of sample collection. The dogs of the healthy dog group had a median weight of $16.5 \mathrm{~kg}$ with a range of $2.6-35.0 \mathrm{~kg}$ and a median age of 5.6 years with a range of $2.0-15.0$ years. The dogs of the diseased dog group had a median weight of $16.0 \mathrm{~kg}$ with a range of $2.5-28.0 \mathrm{~kg}$ and a median age of 7.0 years with a range of 1.0-15.0 years. The healthy dogs were owned by students and staff of Texas A\&M University. At the time of sample collection, all 12 healthy dogs (Table 1) were free from any clinical signs of disease. A complete blood count and serum biochemistry profile were analyzed at the Texas Veterinary Medical Diagnostic Laboratory (College Station, TX). To exclude subclinical gastrointestinal or pancreatic disease in the healthy dogs, serum concentrations of cobalamin and folate (Immulite 2000, Siemens Healthcare Diagnostics Inc., Deerfield, IL, USA), pancreatic lipase immunoreactivity (cPLI; Spec cPL ELISA kit, IDEXX Laboratories, Westbrook, ME, USA), and trypsin-like immunoreactivity (cTLI; I-RIA kit, Siemens Medical Solution Diagnostics, Los Angeles, CA, USA) were measured at the Gastrointestinal Laboratory at Texas A\&M University. Fecal samples from all dogs in the diseased and healthy groups were analyzed for Clostridium perfringens and Clostridium difficile using an enzyme-linked immunosorbent assay kit (C. perfringens Enterotoxin Test and C. difficile Enterotoxin Test, TechLab, Inc., Blacksburg, VA, USA), Giardia and Cryptosporidium using an indirect fluorescent antibody test kit (Merifluor, Meridian Bioscience Inc., Cincinnati, OH), Campylobacter spp. using PCR, and other pathogens using a routine fecal flotation.

One fecal sample per animal was collected immediately after natural defecation, stored at $4^{\circ} \mathrm{C}$ before and during transport to the laboratory (within 24 hours of sample collection), and subsequently stored frozen at $-80^{\circ} \mathrm{C}$ until DNA extraction.

For the diseased dogs, leftover fecal samples that were submitted from veterinary hospitals in the College Station/Houston area for fecal pathogen analysis unrelated to the current study were analyzed. Questionnaires were sent to submitting veterinarians to enquire about the clinical signs of gastrointestinal disease, the duration, and the final diagnosis. Furthermore, to ensure that samples were handled in a similar fashion as the samples from the healthy dogs, information was obtained about the collection, storage, and shipping of samples to the laboratory. Only samples that were handled in a similar fashion to the samples from healthy dogs were analyzed. Leftover fecal samples for the diseased dogs (Table 1) were chosen based on a presenting complaint of acute, uncomplicated, nonhemorrhagic diarrhea (defined as $<72$ hours of onset) that resolved with symptomatic treatment.

At the time of sample collection, none of the evaluated healthy and diseased dogs were receiving any medications expected to alter the gut microbiota (i.e., antibiotics) and were vaccinated and dewormed regularly. All dogs were fed commercial diets and no diet change was reported within the 3-week period prior to sample collection.

This study was approved by the Clinical Research Review Committee of Texas A\&M University (CRRC\#07-38).
2.2. DNA Extraction. Genomic DNA was extracted from each fecal sample using a bead-beating technique followed by phenol-chloroform-isoamyl alcohol extraction as described previously [13].

2.3. 18S rRNA Gene Pyrosequencing for Fungal Organisms (fTEFAP). Tag-encoded FLX-Titanium amplicon pyrosequencing (fTEFAP) and data processing for fungal organisms were performed as described previously $[8,14]$ with panfungal primers forward funSSUF-TGGAGGGCAAGTCTGGTG and reverse funSSUR-TCGGCATAGTTTATGGTTAAG.

The raw data from fTEFAP was screened and trimmed based on quality scores (nominal PHRED20), binned into individual sample collections, and then depleted of any chimeras using B2C2 (http://www.researchandtesting.com/ B2C2.html) [15]. The sequences were then compared against a curated fungal sequence database as reported previously $[8,14]$. Fungal sequences were grouped into operational taxonomic units (OTUs) based on identity scores to known 18 S fungal sequences; $>97 \%$ of identity were reported at the species level, between $95 \%$ and $97 \%$ at the genus level, between $90 \%$ and $95 \%$ at the family level, and between $80 \%$ and $90 \%$ at the order level.

2.4. Statistical Analyses. The pyrosequencing results were expressed as a percentage of the total fungal community in each dog. Only taxa that were present in at least 50\% of dogs (either healthy or diseased) were included in the analysis. All percentage data was tested for normality using the Kolmogorov-Smirnov normality test. As the data was found to be nonparametric, a Mann-Whitney $U$ test was used to compare the percentages of fungal organisms between the healthy and the diseased dogs at the phylum, class, order, family, and genus levels. All statistical analyses were conducted using a commercially available statistical software program (Prism 5, GraphPad, San Diego, CA, USA). Significance level was set at $P<0.05$ for all comparisons.

To visualize differences in the relative abundance of fungal genera in individual samples as a heat map, a double dendrogram was generated using multivariate hierarchical clustering methods based upon Furthest Neighbor metric with Euclidean distances in NCSS 2007 (NCSS, Kaysville, Utah) [16]. The sequencing coverage for each sample was calculated according to Good using the formula $[1-(n / N)]$ $\times 100$, where $n$ is the number of unique sequences and $N$ is the total number of sequences obtained for each sample.

\section{Results}

3.1. Animals. The results of the serum biochemistry profile, complete blood count, serum concentrations of cobalamin, folate, cPLI, and cTLI did not reveal abnormalities in the healthy group. Review of the clinical records of the dogs with acute diarrhea showed that all dogs recovered uneventfully with symptomatic therapy. Results of fecal examination 
TABLE 1: Dogs enrolled into this study.

\begin{tabular}{|c|c|c|c|c|c|}
\hline ID & Health status & Age & Breed & Sex & Weight $(\mathrm{kg})$ \\
\hline $\mathrm{H} 1$ & Healthy & 4.2 & Shih-tzu & fs & 7.0 \\
\hline $\mathrm{H} 2$ & Healthy & 4.0 & English Bulldog & fs & 20.7 \\
\hline H3 & Healthy & 15.0 & Chihuahua & fs & 2.6 \\
\hline $\mathrm{H} 4$ & Healthy & 2.0 & Golden Retriever & $\mathrm{mn}$ & 35.0 \\
\hline H5 & Healthy & 7.8 & Mixed breed & $\mathrm{mn}$ & 30.4 \\
\hline H6 & Healthy & 4.0 & Dachshund & fs & 6.0 \\
\hline $\mathrm{H} 7$ & Healthy & 9.6 & Mixed breed & fs & 32.1 \\
\hline $\mathrm{H} 8$ & Healthy & 10.0 & Mixed breed & $\mathrm{mn}$ & 28.0 \\
\hline $\mathrm{H} 9$ & Healthy & 3.5 & Miniature Schnauzer & $\mathrm{mn}$ & 9.5 \\
\hline $\mathrm{H} 10$ & Healthy & 5.2 & Terrier Mix & $\mathrm{mn}$ & 27.7 \\
\hline H11 & Healthy & 5.9 & Brussels Griffon & $\mathrm{mn}$ & 6.4 \\
\hline $\mathrm{H} 12$ & Healthy & 9.2 & Beagle & $\mathrm{mn}$ & 12.4 \\
\hline D1 & Diseased & 1.5 & Labrador Retriever & fs & 28.0 \\
\hline D2 & Diseased & 11.0 & Mixed Breed & fs & 14.5 \\
\hline D3 & Diseased & 15.0 & Cocker Spaniel & $\mathrm{mn}$ & 16.0 \\
\hline D4 & Diseased & 7.0 & Chihuahua & fs & 2.5 \\
\hline D5 & Diseased & 2.3 & King Charles & fs & 6.0 \\
\hline D6 & Diseased & 14.0 & Golden Retriever & fs & 24.8 \\
\hline D7 & Diseased & 1.0 & Labrador mix & $\mathrm{mn}$ & 18.0 \\
\hline
\end{tabular}

m: male intact; f: female intact; $\mathrm{mn}$ : male neutered; fs: female spayed.

(i.e., fecal flotation, fecal analyses for $C$. perfringens, $C$. difficile, Cryptosporidium, and Giardia, and PCR analysis for Campylobacter spp.) on all dogs did not reveal the presence of any specific enteropathogen.

3.2. Pyrosequencing for Fungal Organisms. A total of 57,179 sequences (median 2800, range 840-6000 per sample) of good quality were obtained. To allow for equal sequencing depth across all samples, 840 randomly selected sequences were analyzed per sample, as described previously [16]. A total of five phyla were identified. Of these, Ascomycota and Basidiomycota were found in $\geq 50 \%$ of dogs in both groups of diseased and healthy dogs. The remaining phyla, Chytridiomycota, Neocallimastigomycota, and Microsporidia, were found in $\leq 50 \%$ of dogs in both groups. A total of 219 fungal genera were identified across all 19 dogs in this study with a median of 28 genera per dog and a range of 4-69 genera per dog. There were a median of 32 genera (range 10$55)$ per dog in the healthy group and a median of 18 genera (range 4-69) per dog in the diseased group. The mean ( \pm SD) coverage was $0.956( \pm 0.0256)$ based on a sequencing depth of 840 sequences per samples.

No significant differences $(P>0.05)$ of the percentage of fungal organisms at the phylum, class, order, family, and genus levels were found between the healthy and diseased dogs. There were no significant differences in age $(P=0.983)$ or weight $(P=0.577)$ between the healthy and diseased groups. Table 2 summarizes the relative percentages in terms of median and range for the most abundant fungal groups on the various phylogenetic levels based on pyrosequencing results (phylum, class, order, and genus levels). Only groups that were present in at least $50 \%$ of either healthy or diseased dogs are shown.

Members of Ascomycota were found in all 19 dogs and this was the most abundant fungal phylum in both the healthy dogs and the diseased dogs with a median of $97.90 \%$ and a range of $63.2 \%-100.0 \%$ and a median of $91.4 \%$ and a range of $91.4 \%-100.0 \%$ of all fungal sequences, respectively. Basidiomycota was the second most abundant fungal phylum with a median of $1.0 \%$ and a range of $0.0-36.8 \%$ for the healthy group and a median of $0.5 \%$ and a range of $0.0 \%-$ $7.8 \%$ for the diseased group. Basidiomycota was found in eight healthy dogs and five diseased dogs.

Within Ascomycota, classes in either the healthy or the diseased groups consisted of Dothideomycetes, Saccharomycetes, Eurotiomycetes, Taphrinomycetes, and Sordariomycetes, with Dothideomycetes being the most abundant class (median: $34.0 \%$, range: $0.3 \%-74.4 \%$ for healthy dogs; median: $28.8 \%$, range: $19.1 \%-90.9 \%$ for diseased dogs). The most prominent orders in Dothideomycetes were Pleosporales (median: $16.1 \%$, range: $0.0 \%-50.9 \%$ for healthy dogs and a median of $15.0 \%$ and a range of $0.0 \%-81.4 \%$ for diseased dogs) and Capnodiales (median: $11.5 \%$, range: $<0.1 \%-36.0 \%$ for healthy dogs; median: $8.7 \%$, range: $0.8 \%-81.8 \%$ for diseased dogs). Pleosporales was the most diverse of the two orders, containing four genera and eight species.

Within Basidiomycota, the predominant classes were Agaricomycetes and Ustilaginomycetes, with the latter consisting almost exclusively the orders Ustilaginales and the genus Ustilaginaceae.

Table 3 shows several species in the major fungal groups identified in the dog feces using pyrosequencing, as well as some species considered to have the potential to be 


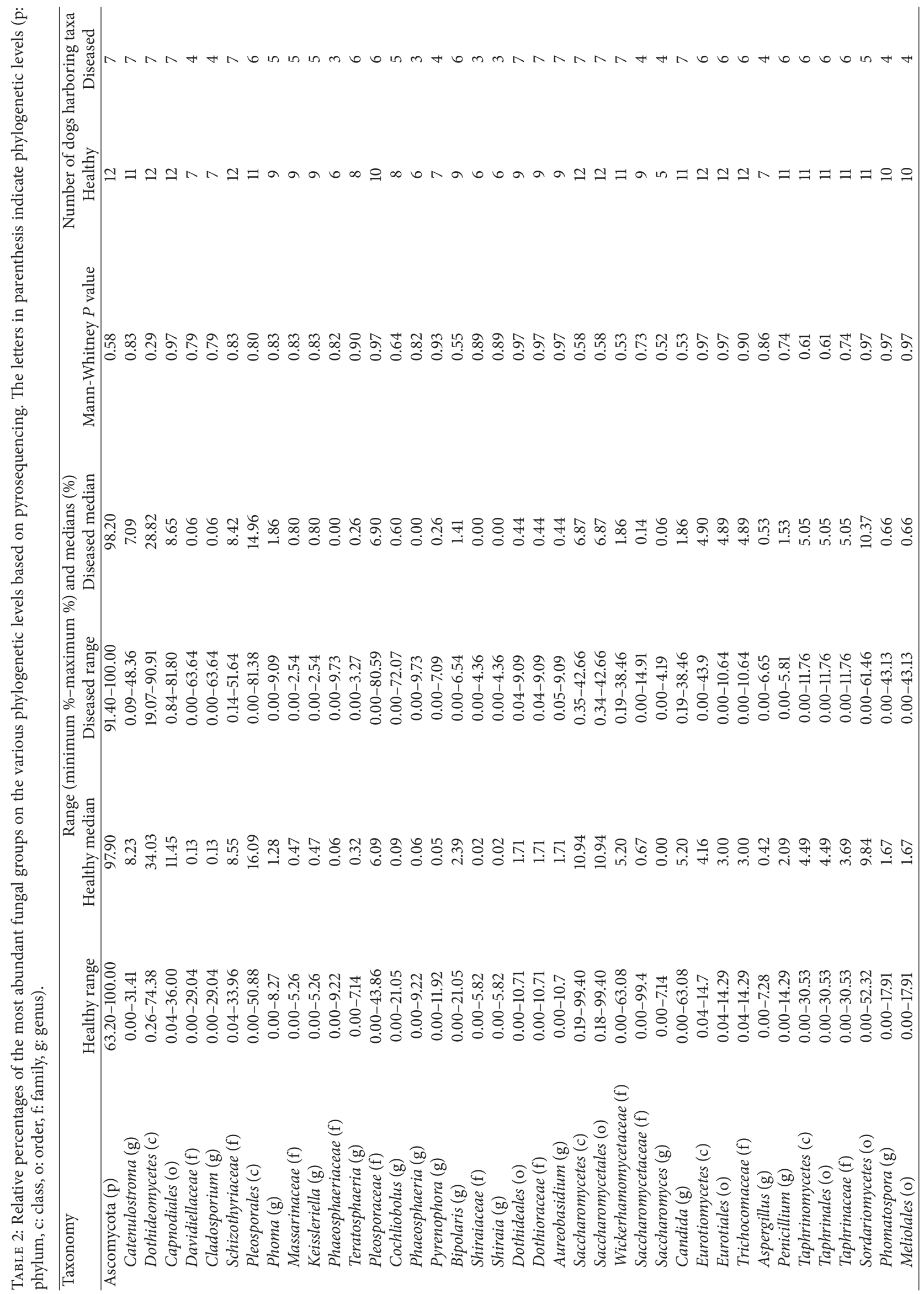




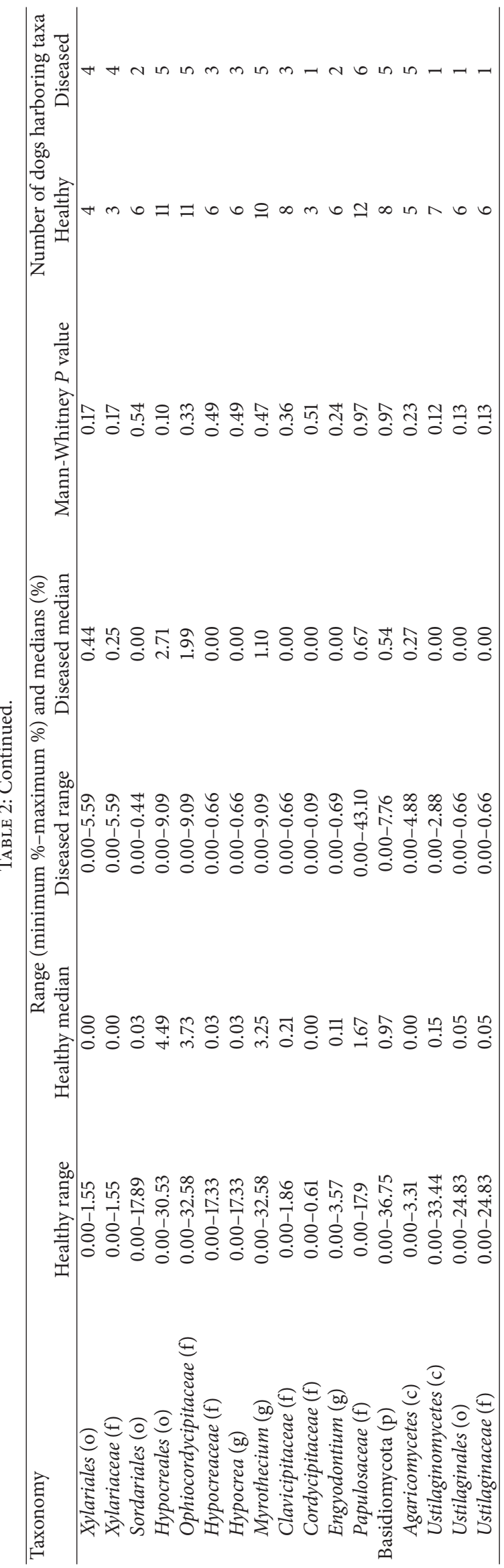



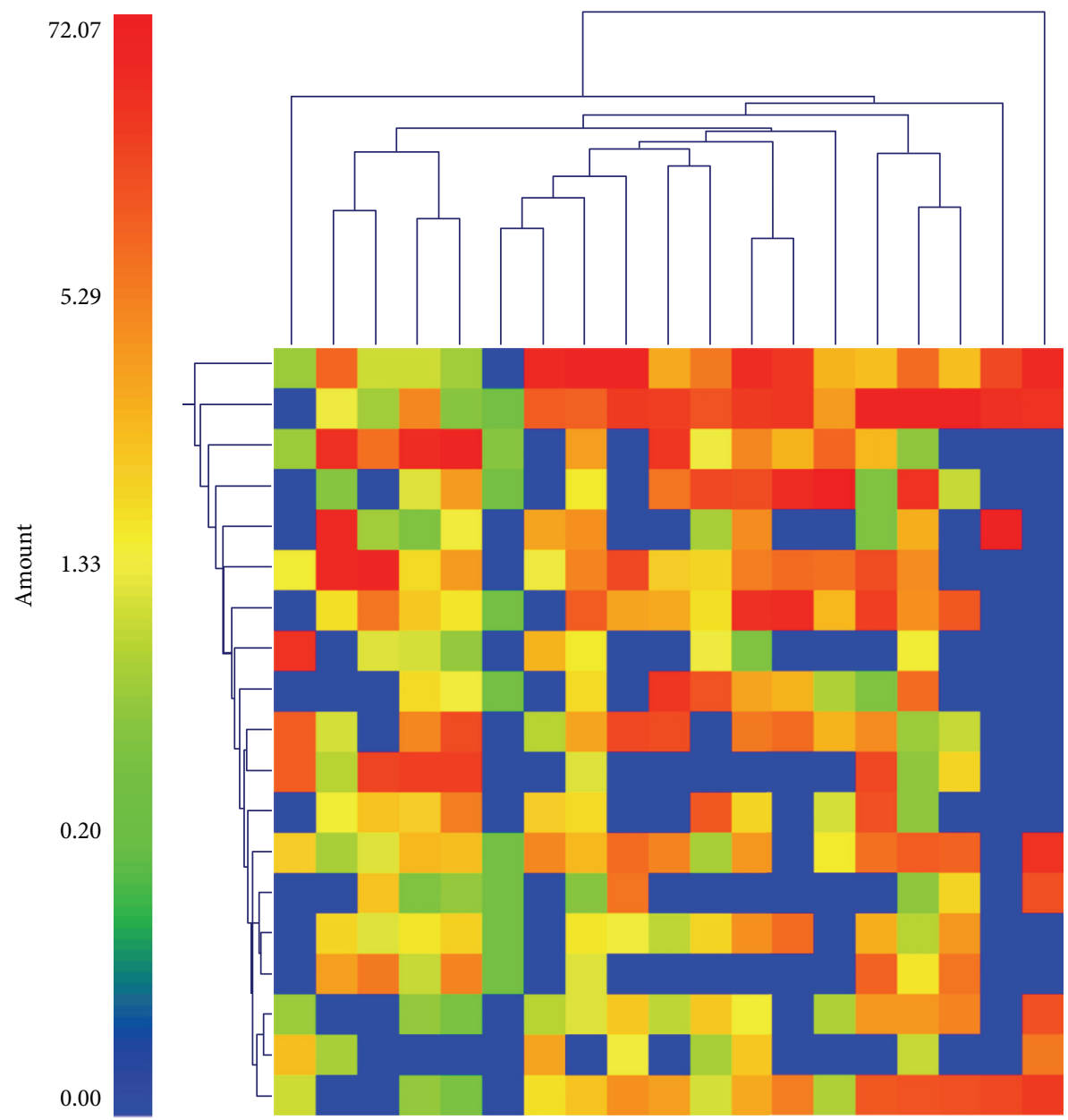

Candida

Catenulostroma

Phomatospora

Cochliobolus

Cladosporium

Myrothecium

Bipolaris

Hypocrea

Pyrenophora

Phoma

Phaeosphaeria

Aspergillus

Penicillium

Saccharomyces

Keissleriella

Shiraia

Teratosphaeria

Engyodontium

H1 H2 H12 D6 D7 H5 H9 H10 D6 H11 D2 H6 H8 D4 H3 H7 D5 D3 H4

Aureobasidium

FIGURE 1: Dual hierarchal dendrogram based upon the predominant fungal genera. The clustering is based upon Furthest Neighbor metric with Euclidean distances. The heat map represents the relative percentages of the most abundant fungal genera identified in each sample $(\mathrm{H}$ $=$ healthy, $\mathrm{D}=$ diseased).

pathogenic. The sequences were blasted against the NCBI database. The accession number, the similarity to their closest neighbor in the NCBI, and the number of healthy and diseased dogs harboring these species are summarized in this table. Figure 1 shows the differences in the relative abundance of the fungal genera in each dog displayed as a heat map.

\section{Discussion}

In this study, 454-pyrosequencing of the fungal $18 \mathrm{~S}$ rRNA gene was utilized to characterize the fungal microbiome present in canine feces. Fecal samples from a total of 19 dogs were individually evaluated. Seven dogs were classified as diseased based on the presence of acute nonhemorrhagic diarrhea, and the remaining 12 were classified as healthy. In the current study, no significant differences in the relative proportions of fungal groups were observed between the diseased and healthy dogs. The most abundant phylum in canine feces was Ascomycota (median: 97.9\%, range: $63.2 \%-$ $100.0 \%$ for healthy and median: $98.2 \%$, range: $91.4 \%-100.0 \%$ for diseased dogs). The most abundant classes included Dothideomycetes and Saccharomycetes, with Candida found to be the most abundant genus.

Previous studies using either culture-based or molecular methods have provided some information about the fungal microbiome present in the GI tract of dogs $[6-9,17]$. Based on cultivation studies, Mentula et al. observed a higher prevalence of yeast in the lumen of the jejunum compared to the feces of healthy dogs (27\% versus 5\%, resp.) [7]. Benno et al. reported the presence of yeasts and molds in the stomach, ileum, colon, and rectum in 2 of 8 healthy Beagle dogs [6]. However, no further specifics regarding the phylogenetic classification of these organisms were reported in these two studies. Using a panfungal PCR, Suchodolski et al. reported that $60 \%$ of healthy dogs and $76 \%$ of dogs with chronic enteropathies were positive for fungal DNA in small intestinal brush samples [10]. All 51 phylotypes identified by Suchodolski et al. were members of the phyla Ascomycota (32 phylotypes) or Basidiomycota (19 phylotypes) [10]. Handl et al. analyzed pooled fecal samples obtained from 12 healthy 
TABLE 3: Fungal species identified in feces from dogs using pyrosequencing. The accession number and similarity to their closest relative in the NCBI database and the number of healthy and diseased dogs harboring the species are summarized.

\begin{tabular}{|c|c|c|c|c|}
\hline Fungal species & Accession no. to closest relative & Similarity & Healthy dogs & Diseased dogs \\
\hline Catenulostroma abietis & FJ267703 & 99 & 11 & 7 \\
\hline Bipolaris eleusines & DQ337382 & 99 & 0 & 1 \\
\hline Bipolaris sorokiniana & DQ337383 & 99 & 8 & 6 \\
\hline Candida albicans & AF114470 & 99 & 0 & 1 \\
\hline Candida austromarina & AB013560 & 99 & 3 & 1 \\
\hline Candida castellii & AY497752 & 92 & 2 & 1 \\
\hline Candida glycerinogenes & AY584809 & 99 & 2 & 0 \\
\hline Candida homilentoma & AB018166 & 99 & 1 & 0 \\
\hline Candida khmerensis & AB158655 & 98 & 1 & 0 \\
\hline Candida mesenterica & AB013552 & 99 & 1 & 0 \\
\hline Candida natalensis & AB013541 & 99 & 10 & 6 \\
\hline Candida neerlandica & EF120593 & 99 & 2 & 0 \\
\hline Candida zeylanoides & EU590665 & 99 & 1 & 0 \\
\hline Aspergillus flavipes & AB002061 & 98 & 0 & 1 \\
\hline Aspergillus niger & JX112703 & 99 & 4 & 3 \\
\hline Aspergillus ochraceus & AB008405 & 99 & 2 & 1 \\
\hline Aspergillus penicillioides & AB002078 & 99 & 4 & 0 \\
\hline Aspergillus terreus & JN639854 & 99 & 5 & 2 \\
\hline Penicillium brevicompactum & AF548082 & 99 & 4 & 0 \\
\hline Penicillium charlesii & FJ430768 & 99 & 4 & 1 \\
\hline Penicillium commune & EU263609 & 99 & 2 & 1 \\
\hline Penicillium coprobium & FJ430772 & 99 & 1 & 0 \\
\hline Penicillium janthinellum & AB293968 & 100 & 7 & 4 \\
\hline Penicillium tardum & AF245233 & 99 & 4 & 1 \\
\hline Penicillium verruculosum & AF510496 & 100 & 4 & 2 \\
\hline Myrothecium cinctum & AJ301996 & 99 & 6 & 5 \\
\hline Myrothecium gramineum & FJ825369 & 99 & 8 & 3 \\
\hline Myrothecium leucotrichum & AJ301992 & 99 & 4 & 2 \\
\hline Cryptococcus gastricus & DQ645513 & 98 & 2 & 0 \\
\hline Cryptococcus surugaensis & $\mathrm{AB} 100440$ & 97 & 1 & 0 \\
\hline
\end{tabular}

dogs using $18 \mathrm{~S}$ rRNA gene pyrosequencing and found that Ascomycota comprised $99 \%$ of the fungal sequences, with Saccharomycetes found to be the most abundant class at $85 \%$ and Candida found to be the most abundant genus [8]. Similarly, using a shotgun DNA sequencing approach, Swanson et al. also identified Ascomycota and Basidiomycota as the most abundant phyla in pooled fecal samples of 6 healthy dogs [9]. However, the overall abundance of fungal sequences was low $(0.01 \%$ of the canine metagenome $)$ and only 3 distinct phylotypes were identified, most likely due to an insufficient sequencing depth [9].

Our results are in general agreement with the previously cited studies. By analyzing the fungal $18 \mathrm{~S}$ rRNA gene in individual fecal samples, we identified a higher number of fungal OTUs (at 97\% similarity; OTU ${ }_{97}$ ) compared to a previous study that used a metagenomic approach based on DNA shotgun sequencing of fecal samples and which reported only 3 OTUs [9]. Also, our study revealed a higher number of OTUs than reported for pooled fecal samples (i.e., $33 \mathrm{OTU}_{97}$ ). This study also suggests that the fecal mycobiome harbors more fungal OTUs compared to the small intestine, as one study examining the small intestinal fungal microbiome reported that $79 \%$ of dogs positive for fungal DNA harbored only one unique phylotype [10]. However, the species richness of fungal organisms appears to be lower compared to the bacterial richness, as several hundred bacterial OTUs have been reported [8] in fecal samples of dogs.

While this study provided insight into the diversity of the fungal microbiome in healthy dogs and dogs with acute diarrhea, some limitations need to be noted. The methods employed did not allow quantitative determination of the fungal abundance. Previous metagenomic studies performed on canine fecal samples suggest that fungal sequences make up a small proportion of the total fecal microbiota with $0.3 \%$ of obtained sequences [9]. Quantitative studies utilizing fluorescence in situ hybridization (FISH) of fungal smallsubunit rRNA probes estimated the abundance of fungi as $<2 \%$ in fecal samples from mice [18] and $<0.03 \%$ in fecal samples from humans [11]. Future studies require the use of a more quantitative enumeration technique such as FISH to 
quantify the abundance of fungi in feces of dogs. The current study was limited in terms of the number of animals and the comparison of a healthy community to a community with only one disease type, and these results were obtained from fecal samples only, not biopsies. The current cost of highthroughput sequencing prohibited the use of a larger sample size.

In conclusion, the current study provides information about the fungal microbiome present in canine feces from both healthy dogs and dogs with acute, nonhemorrhagic diarrhea. The results provide a baseline for future studies evaluating the fungal microbiome in dogs with various gastrointestinal disorders.

\section{Acknowledgment}

This paper was presented in part as an abstract at the Forum of the American College of Veterinary Internal Medicine, Anaheim, California, June 2010.

\section{References}

[1] J. S. Suchodolski, "Companion animals symposium: microbes and gastrointestinal health of dogs and cats," Journal of Animal Science, vol. 89, no. 5, pp. 1520-1530, 2011.

[2] A. S. Neish, "Microbes in gastrointestinal health and disease," Gastroenterology, vol. 136, no. 1, pp. 65-80, 2009.

[3] J. S. Suchodolski, J. F. Garcia-Mazcorro, S. Unterer et al., "The fecal microbiome in dogs with acute diarrhea and idiopathic inflammatory bowel disease," PLoS ONE, vol. 7, no. 12, Article ID e51907, 2012.

[4] J. F. Garcia-Mazcorro, J. S. Suchodolski, K. R. Jones et al., "Effect of the proton pump inhibitor omeprazole on the gastrointestinal bacterial microbiota of healthy dogs," FEMS Microbiology Ecology, vol. 80, no. 3, pp. 624-636, 2012.

[5] J. A. Bell, J. J. Kopper, J. A. Turnbull, N. I. Barbu, A. J. Murphy, and L. S. Mansfield, "Ecological characterization of the colonic microbiota of normal and diarrheic dogs," Interdisciplinary Perspectives on Infectious Diseases, vol. 2008, Article ID 149694, 17 pages, 2008.

[6] Y. Benno, H. Nakao, K. Uchida, and T. Mitsuoka, "Impact of the advances in age on the gastrointestinal microflora of beagle dogs," The Journal of Veterinary Medical Science, vol. 54, no. 4, pp. 703-706, 1992.

[7] S. Mentula, J. Harmoinen, M. Heikkilä et al., "Comparison between cultured small-intestinal and fecal microbiotas in beagle dogs," Applied and Environmental Microbiology, vol. 71, no. 8, pp. 4169-4175, 2005.

[8] S. Handl, S. E. Dowd, J. F. Garcia-Mazcorro, J. M. Steiner, and J. S. Suchodolski, "Massive parallel 16S rRNA gene pyrosequencing reveals highly diverse fecal bacterial and fungal communities in healthy dogs and cats," FEMS Microbiology Ecology, vol. 76, no. 2, pp. 301-310, 2011.

[9] K. S. Swanson, S. E. Dowd, J. S. Suchodolski et al., "Phylogenetic and gene-centric metagenomics of the canine intestinal microbiome reveals similarities with humans and mice," The ISME Journal, vol. 5, no. 4, pp. 639-649, 2011.

[10] J. S. Suchodolski, E. K. Morris, K. Allenspach et al., "Prevalence and identification of fungal DNA in the small intestine of healthy dogs and dogs with chronic enteropathies," Veterinary Microbiology, vol. 132, no. 3-4, pp. 379-388, 2008.
[11] S. J. Ott, T. Kühbacher, M. Musfeldt et al., "Fungi and inflammatory bowel diseases: alterations of composition and diversity," Scandinavian Journal of Gastroenterology, vol. 43, no. 7, pp. 831841, 2008.

[12] T. Kühbacher, S. J. Ott, U. Helwig et al., "Bacterial and fungal microbiota in relation to probiotic therapy (VSL\#3) in pouchitis," Gut, vol. 55, no. 6, pp. 833-841, 2006.

[13] J. S. Suchodolski, C. G. Ruaux, J. M. Steiner, K. Fetz, and D. A. Williams, "Application of molecular fingerprinting for qualitative assessment of small-intestinal bacterial diversity in dogs," Journal of Clinical Microbiology, vol. 42, no. 10, pp. 47024708, 2004.

[14] M. E. Lucero, A. Unc, P. Cooke, S. Dowd, and S. Sun, "Endophyte microbiome diversity in micropropagated Atriplex canescens and Atriplex torreyi var griffithsii," PLoS ONE, vol. 6, no. 3, Article ID e17693, 2011.

[15] V. Gontcharova, E. Youn, R. D. Wolcott, E. B. Hollister, T. J. Gentry, and S. Dowd, "Black box chimera check (B2C2): a windows-based software for batch depletion of chimeras from bacterial 16S rRNA gene datasets," The Open Microbiology Journal, vol. 4, pp. 47-52, 2010.

[16] J. S. Suchodolski, S. E. Dowd, V. Wilke, J. M. Steiner, and A. E. Jergens, "16S rRNA gene pyrosequencing reveals bacterial dysbiosis in the duodenum of dogs with idiopathic inflammatory bowel disease," PLoS ONE, vol. 7, no. 6, Article ID e39333, 2012.

[17] J. S. Suchodolski, J. Camacho, and J. M. Steiner, "Analysis of bacterial diversity in the canine duodenum, jejunum, ileum, and colon by comparative $16 \mathrm{~S}$ rRNA gene analysis," FEMS Microbiology Ecology, vol. 66, no. 3, pp. 567-578, 2008.

[18] A. J. Scupham, L. L. Presley, B. Wei et al., "Abundant and diverse fungal microbiota in the murine intestine," Applied and Environmental Microbiology, vol. 72, no. 1, pp. 793-801, 2006. 

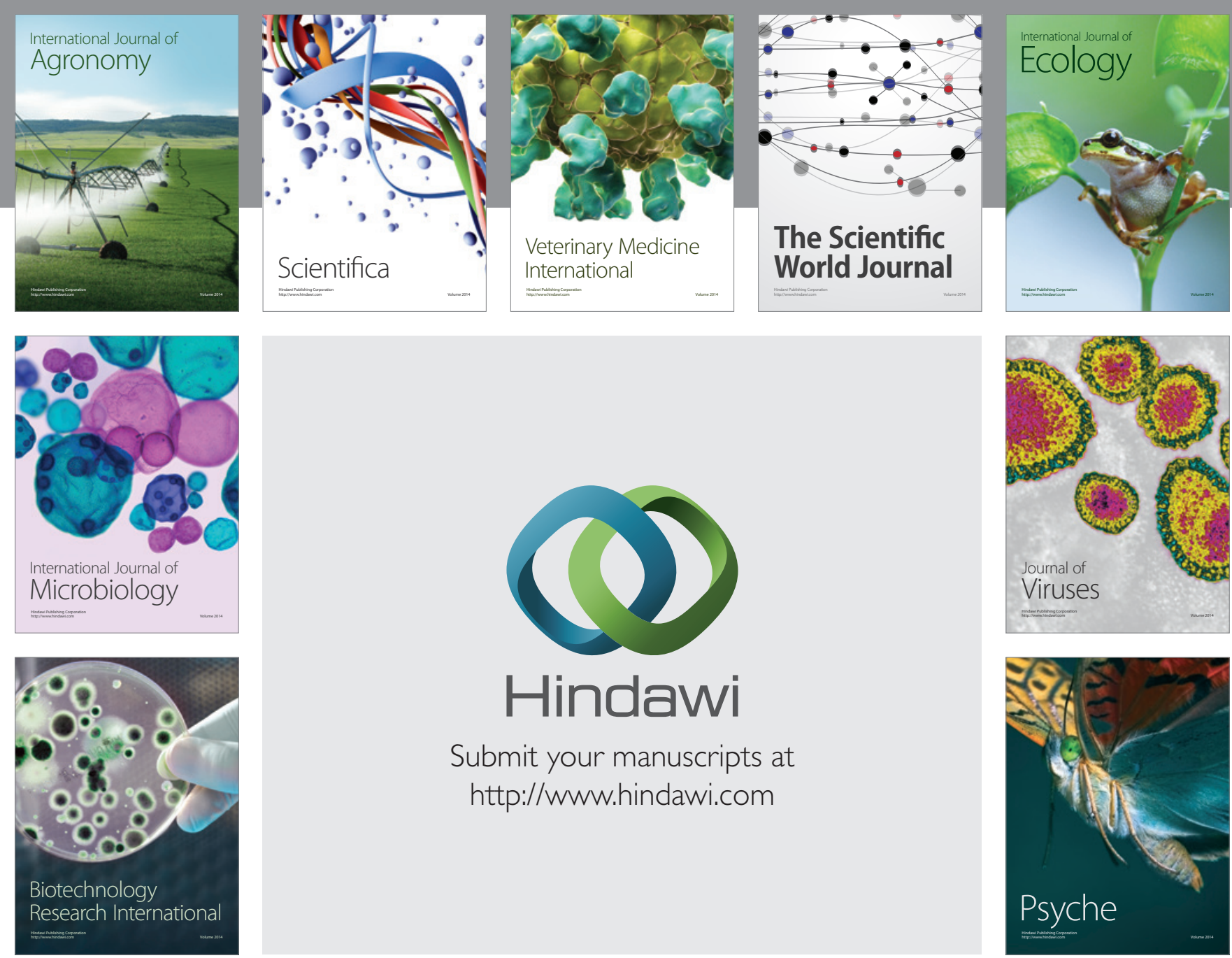

Submit your manuscripts at http://www.hindawi.com
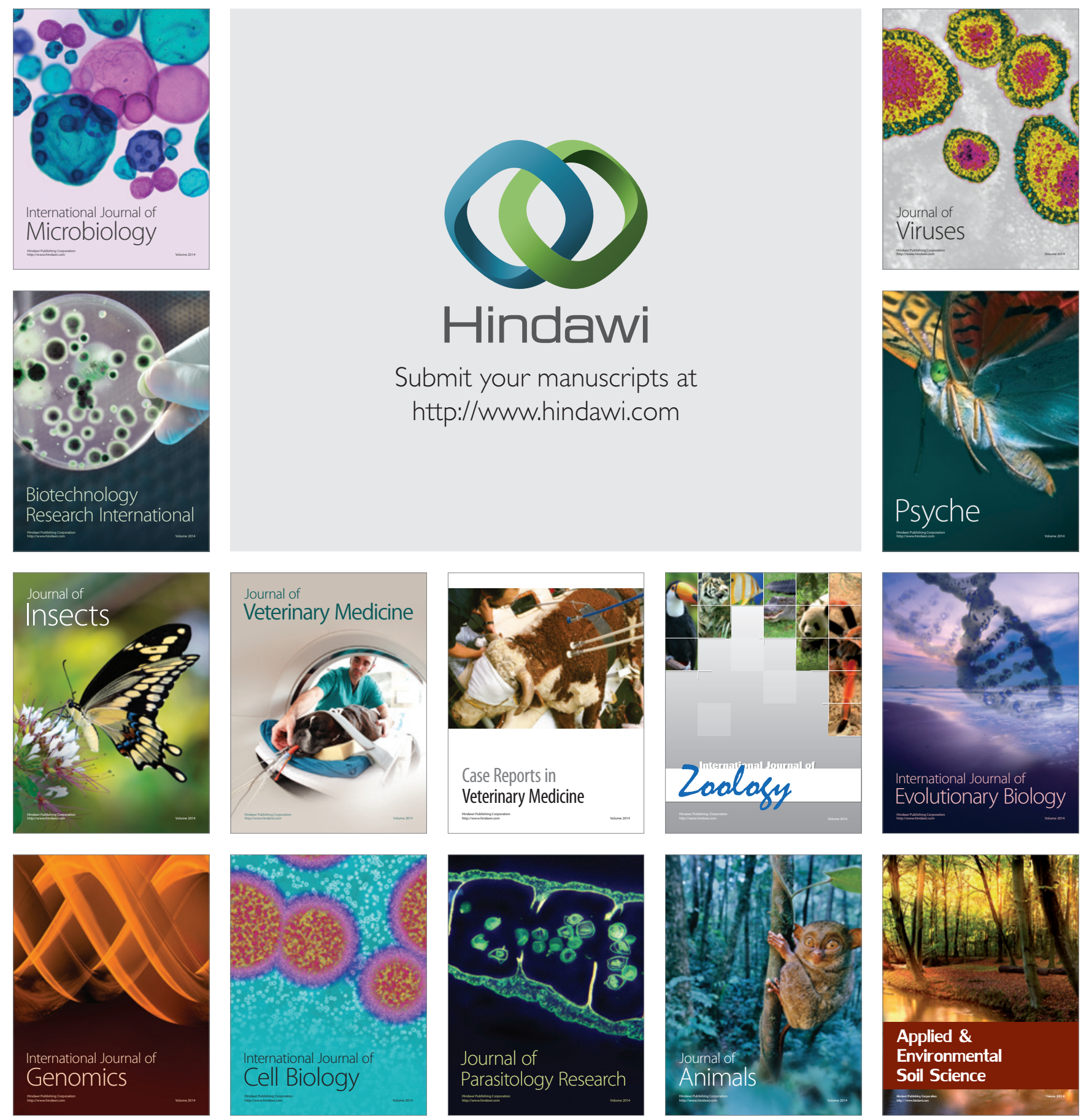